\title{
Notas sobre a socialidade e a biologia de nidificação de Trypoxylon (Trypoxylon) asuncicola Strand, 1910 (Hymenoptera, Sphecidae)
}

\author{
Rui Carlos Peruquetti ${ }^{1,2}$ \\ Marco Antônio Del Lama ${ }^{1}$
}

\begin{abstract}
Notes on the sociality and nesting biology of Trypoxylon (Trypoxylon) asuncicola Strand, 1910 (Hymenoptera, Sphecidae). Twenty-two nests of Trypoxylon asuncicola were sampled in Viçosa, State of Minas Gerais, Brazil, in January 2000 and the occupants' behavior of three nests was registered in $2 \mathrm{~h}$ of direct observation. 528 brood cells were excavated ( $24 \pm 13.84$ SD cells per nest), 129 were reused cells, some of them for seven times (meconium deposit count). The mean number of total cells, mean number of open and closed cells, parasitism rate and mean number of reused cells per nest were similar between old and new nests. Parasitism rate and cell reuse were associated with the number of building cells in the nest, but nest aggregate in the sampled area may play some role in the parasitism rate. Brachymeria sp. (Chalcididae) was the most important agent of brood mortality (80\%). Other parasites were Melittobia sp. (Eulophidae) (17\%) and a species of Icheumonidae (3\%). The number of closed cells with immature individuals per nest was $4 \pm 4.2 \mathrm{SD}(\mathrm{N}=17)$ and the mean reproductivity per female was $3 \pm 2.4 \mathrm{SD}(\mathrm{N}=5)$. New nests produced more offspring $(0$ a $35 \%)$ than old nests $(0$ to $11 \%)$. Females and males can be found resting in the nest but copula or guarding behavior by the male was not observed. There is some evidence that in the sampled area the switch of nests by females is great and agonistic behavior between a nest owner and a visitor was not evident. Females were larger $(3.9 \pm 0.4 \mathrm{SD} \mathrm{mm})$ than males $(3.1 \pm 0.3 \mathrm{SD} \mathrm{mm})$ (measured as forewing length). The secondary sex ratio was $1.26( \pm 0.07 \mathrm{SE})$ in favor of females, which was not different from $1: 1$ ratio. The majority (97\%) of the sampled larvae of $T$. asuncicola showed diapause. Some (5.1\%) 'anomalous cells' were found.
\end{abstract}

KEYWORDs. Nesting biology; parasitism; social behavior; Sphecidae; Trypoxylon.

\section{INTRODUÇÃO}

O gênero Trypoxylon Latreille, 1796 agrupa cerca de 660 espécies (Hanson \& Menke 1995), divididas entre os subgêneros Trypargilum Richards, 1934 (predominantemente Neotropical) e Trypoxylon (cosmopolita). São vespas que usam barro na construção de seus ninhos, feitos em cavidades preexistentes ou livres e que alimentam os imaturos com aranhas paralizadas oferecidas de forma massal. A maioria das espécies é solitária, porém as espécies do grupo albitarse (Trypargilum) podem nidificar em grandes agregados (BRoCKMANN 1992; BRockMANN \& Garfen 1992; Molumby 1995; 1997; CAMILLO 1999) e ninhos comunais (sensu CRESPI \& YANEGA 1995) podem ser encontrados no grupo fabricator (Trypoxylon) (Richards 1934; SAKAgAmi et al. 1990). Neste, a maioria das espécies (sete das 10 reconhecidas) constróem ninhos livres embaixo de folhas de plantas ou em locais protegidos nas construções humanas (RICHARDs 1934; BOHART \& MENKE 1976; SAKAgami et al. 1990). A despeito do grande interesse que as espécies do grupo fabricator despertam naqueles preocupados em entender a evolução do comportamento social em Hymenoptera, apenas T. fabricator F. Smith, 1873 teve sua biologia detalhadamente estudada (SAKAGAMI et al. 1990; GoBBI et al. 1991) e breves relatos sobre a biologia de algumas espécies do grupo são encontrados em RichARDS (1934).

Neste trabalho são reportados alguns aspectos da biologia de Trypoxylon (Trypoxylon) asuncicola Strand, 1910 observados em Viçosa, Minas Gerais.

\section{MATERIALE MÉTODOS}

Os dados apresentados provêm de 22 ninhos coletados nas paredes do Apiário Central da Universidade Federal de

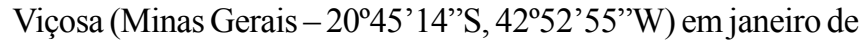

1. Laboratório de Genética Bioquímica, Departamento de Genética e Evolução, Universidade Federal de São Carlos. Caixa Postal 676, 13565-080 São Carlos-SP, Brasil.

2. Endereço atual: Laboratório de Genética Molecular, Departamento de Biologia Geral, Universidade Federal de Viçosa. 36571-000 Viçosa-MG, Brasil. Endereço eletônico: peruquetti@bol.com.br 
2000 e dissecados sob estereomicroscópio no Laboratório de Genética Bioquímica da Universidade Federal de São Carlos (São Paulo). Foram coletados 72 imaturos que foram mantidos, até a emergência dos adultos, individualizados em frascos de vidro tampados com chumaço de algodão e forrados com papel filtro, deixados em local escuro e à temperatura ambiente. Também são apresentados resultados de $2 \mathrm{~h}$ consecutivas de observação, feita no local de nidificação, em três dos 22 ninhos amostrados. Como os adultos passam a noite no ninho, todos os 22 ninhos foram coletados neste período.

Em Apoidea, o comprimento da asa anterior (base da tégula até a ponta da asa) é um bom estimador do tamanho do indivíduo (Coville \& Griswold 1983; Rust 1991; Molumby 1997). Assim, esta medida foi tomada, com auxílio de estereomicroscópio e ocular micrométrica (precisão de $0,05 \mathrm{~mm}$ ), nos machos e fêmeas que emergiram no laboratório para verificar possíveis diferenças de tamanho entre os sexos.

Os testes estatísticos empregados estão de acordo com ZAR (1999). Espécimes-testemunha foram depositados no Museu de Zoologia, Universidade de São Paulo, São Paulo (MZSP).

\section{RESULTADOS E DISCUSSÃO}

A arquitetura do ninho de $T$. asuncicola é semelhante à de outros representantes do grupo fabricator que constróem ninhos livres. Uma revisão sobre o assunto pode ser vista $\mathrm{em}$ RichARDS (1934) e SAKAGAMI et al. (1990). Em média, os ninhos de $T$. asuncicola apresentaram 24 células de cria construídas ( \pm 13 ,84 DP; mínimo, 7; máximo, 49). Ao todo, foram amostradas 528 células, sendo $129(24,4 \%$ ) células reutilizadas (Tabela I). A freqüência de reutilização variou de zero a sete (moda 2), valores obtidos contando-se as deposições de mecônio existentes no fundo de cada célula de cria (Tabela I). Neste aspecto, $T$. asuncicola difere de T. fabricator. SAKAGAMI et al. (1990) relatam que as células de cria de $T$. fabricator são totalmente limpas pelos adultos, exceto a seda presa às paredes da célula.

A reutilização do ninho pode assumir papel importante na evolução de sistemas sociais (MICHENER 1974), principalmente de sistemas menos avançados. Estes poderiam ser formados de duas maneiras: 1) pela permanência de duas ou mais fêmeas no ninho materno ou 2) pela associação de uma ou mais fêmeas a ninhos já estabelecidos por uma única fêmea. Aparentemente, é possível que os dois tipos de reutilização de ninhos ocorram em T. asuncicola e em outras espécies de Trypoxylon do grupo fabricator (SAKAGAMI et al. 1990), com algumas diferenças na reutilização das células de cria.

Entre os ninhos amostrados, 11 apresentaram células fechadas estocadas com aranhas mas sem imaturos, ou células abertas com aranhas secas. Um ninho (ap016) tinha uma célula fechada sem nada em seu interior. SAKAGAMI et al. (1990) relatam a ocorrência de 'células de cria anômalas' em T. fabricator e elas também são encontradas em Trypoxylon (Trypoxylon) tenoctilan Richards, 1934 (Coville \& Coville 1980) e em algumas espécies de Trypargilum (Coville 1981). A razão destas células é desconhecida, mas certamente representam perdas consideráveis da capacidade reprodutiva das fêmeas, pois a coleta de material para nidificação requer muita energia e a cada saída da fêmea do ninho há risco de predação, além de riscos associados à cópula e a interações com outras fêmeas (HunTER et al. 1993).

Dos 22 ninhos amostrados, 10 apresentavam sinais de ação do tempo (erosão das paredes das células de cria mais externas e teias de aranha presas a elas). Estes ninhos foram classificados como velhos, apesar de dois deles serem ocupados por adultos (machos e fêmeas) e cinco ainda possuirem células com imaturos (Tabela I). Provavelmente, estes ninhos representam a fase final do ciclo reprodutivo destas vespas (GoвB et al. 1991; veja também os dados de produtividade abaixo). Os outros 12, não apresentavam estes sinais e foram classificados como novos. Os ninhos velhos não apresentaram maior número de células de cria (mínimo 8; máximo 35) do que os ninhos novos (mínimo 7; máximo 49) $\left(\mathrm{t}_{0,05 ; 20}=1,43 ; \mathrm{P}=0,17\right)$, nem maior taxa de parasitismo (teste exato de Fisher, $\mathrm{P}=0,62$ ) ou maior número de células reutilizadas $\left(\mathrm{t}_{0,05 ; 20}=0,53 ; \mathrm{P}=0,60\right)$. Os ninhos velhos também não foram estatisticamente diferentes dos novos em relação ao número de células abertas (desocupadas ou em construção) ou fechadas (ocupadas) (respectivamente, $\mathrm{t}_{0,05 ; 20}$ $=0,59$ e $_{0,05 ; 20}=1,73 ; \mathrm{P}>0,05$ em ambas as análises). Assim, é possível que os ninhos de $T$. asuncicola tenham crescimento lento ao longo do tempo e as maiores taxas de incremento no número de células aconteça no início do processo de nidificação, como observado em outras vespas e abelhas comunais com fundação solitária de ninhos (haplometrose) (Wilson 1971; TuRILlaZZI 1991; GianNOTTI 1998).

Houve associação entre o número de células de cria do ninho, a taxa de reutilização das células e a taxa de parasitismo (coeficente de concordância de Kendall, $\mathrm{W}=0,75, \times \chi_{0,05 ; 21}^{2}=$ 47,15; $\mathrm{P}<0,001)$. Aparentemente, o número de células construídas no ninho influencia as taxas de reutilização de células e de parasitismo (correlação de Pearson; $n^{\circ}$ de células vs. reutilização; $\mathrm{r}=0,62 ; \mathrm{P}<0,01 ; \mathrm{n}^{\circ}$ de células vs. parasitismo: $\mathrm{r}=0,76 ; \mathrm{P}<0,0001$; reutilização vs. parasitismo: $\mathrm{r}=0,33 ; \mathrm{P}=$ 0,$13 ; \mathrm{N}=22$ para todos os casos). Estes resultados sugerem que cada ninho de $T$. asuncicola tem longa duração, sendo reutilizado por várias gerações e esta persistência do ninho, associada ao hábito gregário de nidificação existente em muitos Sphecidae, favoreceria o aumento das populações dos parasitas (RosenHEIM 1990; WCISLO 1996).

Em média, foram encontradas $4 \pm 4,2 \mathrm{DP}(\mathrm{N}=17)$ células com imaturos por ninho. De apenas $10 \%$ das células emergiram adultos. Considerando-se apenas os indivíduos emergidos, a produtividade média das fêmeas $(\mathrm{N}=5)$ foi de $3 \pm 2,4 \mathrm{DP}$. Os ninhos ativos tiveram maior taxa de emergência (0 a 35\%) do que os ninhos inativos (0 a 11\%) (dados transformados: arcoseno; $\left.\mathrm{t}_{0,05 ; 20}=3,30 ; \mathrm{P}<0,01\right)$. Porém, esta taxa não foi função do número de células presentes no ninho $[\mathrm{y}=0,44 \mathrm{x}-$ 0,$12 ; \mathrm{F}_{1,11}=1,29 ; \mathrm{P}>0,05$; dados transformados: $\left.\operatorname{Ln}(\mathrm{x}+1)\right]$. Estes valores de produtividade são relativamente baixos quando comparados aos das espécies solitárias de Trypoxylon (CAmillo et al. 1993, 1994), mas estão de acordo com o esperado para himenópteros comunais (KUKUK \& SAGE 1994; SANTOS \& 
Tabela I. Dados sobre ninhos de Trypoxylon asuncicola Strand, 1910 coletados em Viçosa, MG, em janeiro de 2000 . ? = sexo não determinado; F = fêmea, $\mathrm{M}=$ macho. 0 = ninhos velhos; 1 = ninhos novos.

\begin{tabular}{|c|c|c|c|c|c|c|c|c|c|}
\hline Ninho & $\begin{array}{l}\text { Adultos } \\
\text { no ninho }\end{array}$ & $\begin{array}{l}\mathrm{N}^{\mathrm{o}} \text {. de } \\
\text { células }\end{array}$ & $\begin{array}{r}\text { Células } \\
\text { abertas }\end{array}$ & $\begin{array}{c}\text { Células } \\
\text { fechadas }\end{array}$ & $\begin{array}{c}\text { Células } \\
\text { reutilizadas }\end{array}$ & $\begin{array}{c}\text { Células com } \\
\text { provisão } \\
\text { sem imaturos } \\
\end{array}$ & $\begin{array}{c}\text { Células } \\
\text { parasitadas }\end{array}$ & Emergidos & $\begin{array}{l}\text { Condição } \\
\text { do ninho }\end{array}$ \\
\hline ap001 & $2(?)$ & 13 & 3 & 10 & 2 & & 7 & $2(1 \mathrm{~F}: 1 \mathrm{M})$ & 1 \\
\hline ap002 & & 12 & 7 & 5 & 4 & & & & 0 \\
\hline Ap003 & & 8 & 1 & 7 & 3 & & & & 0 \\
\hline ap004 & & 9 & 2 & 7 & & & 1 & $1(\mathrm{M})$ & 0 \\
\hline ap005 & $1(\mathrm{~F})$ & 19 & 3 & 16 & 2 & 5 & 1 & $1(\mathrm{~F})$ & 1 \\
\hline ap006 & & 49 & 9 & 40 & 9 & 6 & 11 & & 1 \\
\hline ap007 & & 12 & 4 & 8 & & & 3 & $1(\mathrm{~F})$ & 0 \\
\hline ap008 & & 17 & 1 & 16 & 3 & 2 & & $1(\mathrm{~F})$ & 0 \\
\hline ap009 & $3(2 \mathrm{~F}, 1 \mathrm{M})$ & 34 & 9 & 25 & 16 & 2 & 10 & $10(4 \mathrm{~F}: 6 \mathrm{M})$ & 1 \\
\hline ap010 & & 12 & 3 & 9 & 1 & & 3 & & 0 \\
\hline ap011 & & 11 & & 11 & 3 & & & $1(\mathrm{M})$ & 1 \\
\hline ap012 & & 35 & 6 & 29 & 25 & 2 & 2 & $2(1 \mathrm{~F}: 1 \mathrm{M})$ & 0 \\
\hline ap013 & & 42 & 1 & 41 & 6 & & 17 & $2(1 \mathrm{~F}: 1 \mathrm{M})$ & 1 \\
\hline ap014 & & 7 & 3 & 4 & & 2 & & $1(\mathrm{~F})$ & 1 \\
\hline ap015 & $1(\mathrm{M})$ & 43 & 1 & 42 & 28 & 2 & 14 & $15(7 \mathrm{~F}: 8 \mathrm{M})$ & 1 \\
\hline ap016 & $1(\mathrm{~F})$ & 35 & 3 & 32 & 9 & 3 & 5 & $4(3 \mathrm{~F}: 1 \mathrm{M})$ & 1 \\
\hline ap017 & $3(?)^{*}$ & 38 & 5 & 33 & 2 & 1 & 16 & $3(\mathrm{~F})$ & 1 \\
\hline ap018 & & 25 & & 25 & 4 & 1 & 2 & $1(\mathrm{~F})$ & 0 \\
\hline ap019 & $2(1 \mathrm{~F}, 1 \mathrm{M})$ & 32 & 9 & 23 & 4 & 1 & 14 & & 0 \\
\hline ap020 & & 23 & 9 & 14 & 1 & & 2 & $4(2 \mathrm{~F}: 2 \mathrm{M})$ & 1 \\
\hline ap021 & & 17 & 1 & 16 & 2 & & 6 & $3(2 \mathrm{~F}: 1 \mathrm{M})$ & 1 \\
\hline ap022 & $2(1 ?, 1 \mathrm{M})$ & 35 & 12 & 23 & 5 & & 4 & & 0 \\
\hline TOTAL & $5 \mathrm{~F}, 4 \mathrm{M}, 6 ?)$ & 528 & 92 & 436 & 129 & 27 & 118 & $52(29 \mathrm{~F}: 23 \mathrm{M})$ & $1=12 ; 0=10$ \\
\hline
\end{tabular}

* Ao menos um dos indivíduos era fêmea.

Garófalo 1994). Aparentemente, a baixa produtividade da colônia e/ou de seus ocupantes pode ser contornada pela possibilidade de controle mais efetivo de algumas condições ambientais, principalmente parasitas e predadores (MICHENER 1974; WCISLO 1984; EDGERLY 1994), cujas pressões podem variar muito espacial e temporalmente, mas que são as principais responsáveis pelo insucesso na nidificação de muitas espécies de vespas e abelhas (STRASSAMANN et al. 1988; ITô 1993; WCISLO 1996). Em agregados de ninhos, a ação de parasitas e predadores pode ser devastadora, impedindo o sucesso reprodutivo da maioria das fêmeas do agregado (MichENER 1974; WCISLO et al. 1985; Molumby 1995).

A maioria $(77,3 \%)$ dos ninhos amostrados tinha parasitas. O parasitismo por Brachymeria sp. (Chalcididae) foi o maior responsável pela mortalidade de imaturos nos ninhos amostrados (80\%). Outros parasitas encontrados foram Melittobia sp. (Eulophidae; 17\%) e um Ichneumonidae (3\%). Em T. fabricator, SAKAGAMI et al. (1990) relatam taxas de parasitismo de $44 \%$, sendo $32 \%$ causada por um Chalcididae.

Três ninhos (ap015, ap016 e ap017) foram observados e pode-se verificar o seguinte: os ninhos estavam muito próximos uns dos outros (distância ap015 - ap016: 25 cm; ap015 - ap017:
$48 \mathrm{~cm}$; ap016 - ap017: $32 \mathrm{~cm}$ ); o ap017 apresentava três adultos (o sexo dos indivíduos não pode ser determinado, mas ao menos um era fêmea), o ap015, um macho e o ap016, uma fêmea; o ap015 apresentava 43 células de cria, o ap016, 35 e o ap017, 38 (Tabela I). Um indivíduo permaneceu todo o tempo no ap017, ocupando uma única célula. Os outros dois indivíduos desse ninho, entretanto, o deixavam com bastante freqüência. Apenas uma única vez um deles retornou com uma presa; após várias tentativas de estocá-la em algumas células abertas conseguiu fazê-lo em uma. Por várias vezes, os dois adultos do ap017, ao deixarem o ninho, iam ora para o ap015 (com apenas uma célula vazia, sempre ocupada por um indivíduo), ora para o ap016 (com três células vazias, uma sempre ocupada por um indivíduo). Nestas visitas não foram observadas interações agonísticas entre os ocupantes dos ninhos e os visitantes. Por várias vezes, os visitantes inspecionaram células vazias do ap016, mas a natureza dessas inspeções não foi determinada.

Tolerância a co-específicos pode ser favorável do ponto de vista da evolução do comportamento social, já que permite a união e cooperação entre fêmeas, principalmente na fase inicial de nidificação, ou mesmo surgimento de dominância reprodutiva entre as fêmeas. Esta dominância pode ser 
conseguida via exploração do trabalho das fêmeas associadas, como roubo de material de construção e provisões, oofagia, diminuição do risco de predação para a fêmea dominante que deixa menos o ninho e tem seu vigor e potencial reprodutivo aumentado (ZuCCHI et al. 1969; MiCHENER 1974; RöSELER 1991; KuKuK 1992). Por outro lado, a usurpação do ninho também pode ser favorecida com o aumento da tolerância a coespecíficos (FIELd \& Foster 1995). Também é interessante notar que em outras espécies comunais, a troca de ninhos pelas fêmeas pode ocorrer em taxas elevadas, sem aparente diminuição do potencial reprodutivo das fêmeas associadas (ABRAMS \& EICKWORT 1981; McCorquOdAle 1989).

A presença de machos em ninhos ativos de Trypoxylon (Trypoxylon) não é comum, mas foi registrada em T. fabricator (RichARDS 1934; SAKAGAMI et al. 1990). Estes machos, assim como em $T$. asuncicola, aparentemente, não atuam como guardas, como acontece em Trypoxylon (Trypargilum) (Coville 1981).

As medidas do comprimento das asas anteriores de machos e fêmeas emergidos no laboratório mostraram que os machos são menores $(3,1 \pm 0,3 \mathrm{DP} \mathrm{mm} ; \mathrm{N}=16)$ do que as fêmeas $(3,9 \pm 0,4$ $\mathrm{DP}$ mm; $\mathrm{N}=26)\left(\mathrm{t}_{0,05 ; 40}=6,6 ; \mathrm{P}<0,001\right)$. Dimorfismo sexual em relação ao tamanho do corpo, sendo as fêmeas maiores do que os machos, é comum em Trypoxylon (COVILLE \& GRISWOLD 1983; Assís et al. 1994; MolumBy 1997; SAKAGAMi et al. 1990).

A razão sexual secundária (29 F:23 M ou 1,26 $\pm 0,07$ EP) não foi estatisticamente diferente de $1: 1\left(\chi_{0,05 ; 1}^{2}=0,69 ; \mathrm{P}>0,20\right)$. Estes dados são muito preliminares para serem discutidos, mas em $T$. fabricator a razão sexual é a favor de fêmeas (SAKAGAMi et al. 1990).

A maioria (97\%) das larvas amostradas estava em diapausa. O tempo de permanência destas larvas no laboratório até a emergência dos adultos foi muito variável (37,7 $\pm 34,75$ DP dias; $\mathrm{N}=41$; mínimo, 1; máximo, 119 dias). Esta variação também é observada dentro dos ninhos. Por exemplo, para o ap015 passaram-se 115 dias entre a emergência do primeiro e do último adulto. A diapausa em insetos é geneticamente programada e condicionada por estímulos ambientais, como fotoperíodo, temperatura e disponibilidade de alimento (HARVEY 1962; TAuber \& TAuber 1976). Sob condições desfavoráveis, a diapausa tem início, sendo interrompida quando as condições ideais para reprodução são restabelecidas (CROss et al. 1975; STRAND 1986; KNERER 1993). Não foi possível apontar o fator promotor da diapausa em $T$. asuncicola, mas há um aspecto interessante a ser ressaltado. Em T. asuncicola são encontradas, em taxas elevadas, larvas em diapausa ao longo de todo o ano (L. F. Gomes, comunicação pessoal) e, aparentemente, ela ocorre sem sincronismo entre os ninhos da área ou mesmo entre os indivíduos de um mesmo ninho. A diapausa, além de possibilitar que os adultos sejam ativos em períodos mais favoráveis à sua reprodução (KNERER 1993), também permite que parte da população escape da ação de parasitas (WILSON 1971) podendo, ainda, promover o aparecimento de sistemas sociais devido a possíveis flutuações demográficas dos sexos ao longo do tempo (SEGER 1983; BrockMANN \& GRAFEN 1992; YANEGA 1997).
Agradecimentos. À FAPESP pelo auxílio financeiro (Processo no. 98/16390-0). A Lucio A. O. Campos pelo apoio, Sérvio T. P. Amarante pela identificação de Trypoxylon asuncicola, Marcelo Tavares pela identificação de Brachymeria sp. e aos consultores anônimos da RBE pelas sugestões.

\section{REFERÊNCIAS}

Abrams, J. \& G. C. Eickwort. 1981. Nest switching and guarding by the communal sweat bee Agaspostemon virescens (Hymenoptera, Halictidae). Insectes Sociaux 28(2): 105-116.

Asís, J. D.; J. Tormos \& S. F. Gayubo. 1994. Biological observations on Trypoxylon attenuatum and description of its mature larva and its natural enemy Trichrysis cyanea (Hymenoptera Sphecidae, Chrysididae). Journal of the Kansas Entomological Society 67(2): 199-207.

Bohart, R. M. \& A. S. Menke. 1976. Sphecide wasps of the world. A generic revision. Berkeley, University of California Press, $695 \mathrm{p}$.

Brockmann, H. J. 1992. Male behavior, courtship and nesting in Trypoxylon (Trypargilum) monteverdeae (Hymenoptera: Sphecidae). Journal of the Kansas Entomological Society 65(1): 66-84.

Brockmann, H. J. \& A. Grafen. 1992. Sex ratios and life-history patterns of a solitary wasp, Trypoxylon (Trypargilum) politum (Hymenoptera: Sphecidae). Behavioral Ecology and Sociobiology 30: 7-27.

CAmillo, E. 1999. Bionomics of Trypoxylon (Tryipagilum) antropovi and Trypoxilon (Trypargilum) aestivale (Hymenoptera, Sphecidae). Inheringia, Série Zoologia, 87: 29-36.

Camillo, E.; C. A. Garófalo; G. Muccillo \& J. C. Serrano. 1993. Biological observation on Trypoxylon (Trypargilum) lactitarse Saussure in Southeastern Brazil (Hymenoptera, Sphecidae). Revista Brasileira de Entomologia 37: 769-778.

Camillo, E.; C. A. Garófalo \& J. C. Serrano. 1994. Observações sobre a biologia de Trypoxylon (Trypargilum) rogenhoferi Kohl (Hymenoptera: Sphecidae). Anais da Sociedade Entomológica do Brasil 23: 299-310.

Coville, R. E. 1981. Biological observations on three Trypoxylon wasps in the subgenus Trypargilum from Costa Rica: T. nitidum schulthessi, $T$. saussurei, and T. lactitarse (Hymenoptera: Sphecidae). Pan-Pacific Entomologist 57(2): 332-340.

Coville, R. E. \& C. Griswold. 1983. Nesting biology of Trypoxylon xanthandrum in Costa Rica with observation on its spider prey (Hymenoptera: Sphecidae; Araneae: Senoculidae). Journal of the Kansas Entomological Society 56(2): 205-216.

Coville, R. E. \& P. L. Coville. 1980. Nesting biology and male behavior of Trypoxylon (Trypargilum) tenoctitlan in Costa Rica (Hymenoptera: Sphecidae). Annals of the Entomological Society of America 73(1): 110-119.

Crespi, B. J. \& D. Yanega. 1995. The definition of eusociality. Behavioral Ecology 6: 109-115.

Cross, E. A.; M. G. Stith \& T. R. Bauman. 1975. Bionomics of the organpipe mud-dauber, Trypoxylon politum (Hymenoptera: Sphecidae). Annals of the Entomological Society of America 68(5): 901 916.

Edgerly, J. S. 1994. Is group living an antipredator defense in a facultatively communal webspinner (Embiidina: Clothodidae)? Journal of Insect Behavior 7(2): 135-147.

Field, J. \& W. A. Foster. 1995. Nest co-occupation in the digger wasp Cerceris arenaria: cooperation or usurpation? Animal Behaviour 50: $99-112$.

Giannotti, E. 1998. The colony cycle of the social wasp, Mischocyttarus cerberus styx Richards, 1940 (Hymenoptera, Vespidae). Revista Brasileira de Entomologia 41(2-4): 217-224.

GoBBi, N.; S. F. SAKAGAMI \& R. ZuCCHI. 1991. Nesting biology of a quasisocial sphecid wasp Trypoxylon fabricator II. Life history of nests and adult behavior on nests. Japanese Journal of Entomology 59(1): 37-51.

Hanson, P. E. \& A. S. Menke. 1995. The sphecid wasps (Sphecidae), p. 621-649. In: P. E. Hanson \& I. A. Gauld (eds.). The Hymenoptera 
of Costa Rica. New York, Oxford University Press, 893 p.

Harvey, W. R. 1962. Metabolic aspects of insects diapause. Annual Review of Entomology 7: 57-80

Hunter, F. M.; M. Petrie; M. Otronen; T. Birkhead \& A. P. Moller. 1993. Why do females copulate repeatedly with one male? Trends in Ecology and Evolution 8(1): 21-26.

ITô, Y. 1993. Behaviour and Social evolution of wasps. The communal aggregation hypothesis. (Oxford Series in Ecology and Evolution).Oxford, Oxford University Press, 159 p.

KNERER, G. 1993. Life history diversity in sawflies. p. 33-59. In: M. WAGner \& K. F. Raffa (eds.). Sawfly life history adaptations to woody plants. San Diego, Academic Press, 581p.

KukuK, P. F. 1992. Cannibalism in social bees. p. 214-237. In: M. A. Elgar \& B. J. Crespi (eds.). Ecology and Evolution among diverse taxa. Oxford, Oxford University Press, $547 \mathrm{p}$.

KuKuK, P. F. \& G. K. SAGE. 1994. Reproductivity and relatedness in a communal halictine bee Lasioglossum (Chialictus) hemichalceum. Insectes Sociaux 41: 443-455.

McCorquodale, D. B. 1989. Nest sharing, nest switching, longevity and overlap of generations in Cerceris antipodes (Hymenoptera: Sphecidae). Insectes Sociaux 36(1): 42-50.

Michener, C. D. 1974. The social behaviour of the bees. A comparative study. Cambridge, Belknap Press, $404 \mathrm{p}$.

Molumby, A. 1995. Dynamics of parasitism in the organ-pipe wasp, Trypoxylon politum: effects of spatial scale on parasitoid functional response. Ecological Entomology 20: 159-168.

Molumby, A. 1997. Why make daughter larger? Maternal sex-allocation and sex-dependent selection for body size in a mass-provisioning wasp, Trypoxylon politum. Behavioral Ecology 8(3): 279-287.

Richards, O. W. 1934. The American species of the genus Trypoxylon. Transactions of the Royal Entomological Society of London 82: $173-362$.

RÖSELER P. F. 1991. Reproductive competition during colony establishment p. 309-335. In: K. G. Ross \& R. W. Matthews, (eds.). The social biology of wasps. Ithaca, Cornell University Press, $678 \mathrm{p}$.

Rosenheim, J. A. 1990. Density-dependent parasitism and the evolution of aggregated nesting in the solitary Hymenoptera. Annals of the Entomological Society of America 83(3): 277-286.

Rust, R. W. 1991. Size-weight relationship in Osmia lignaria propinqua Cresson (Hymenoptera: Megachilidae). Journal of the Kansas Entomological Society 64(2): 174-178.

SAKAGAMI, S. F.; N. GobBi \& R. ZuCCHI. 1990. Nesting biology of a quasisocial sphecid wasp Trypoxylon fabricator I. Nests and Inhabitants.
Japanese Journal of Entomology 58(4): 846-862.

Santos, M. L. \& C. A. Garófalo. 1994. Nesting biology and nest re-use of Eulaema nigrita (Hymenoptera: Apidae, Euglossini). Insectes Sociaux 41: 99-110.

SEgER, J. 1983. Partial bivoltinism may cause alternating sex-ratio biases that favour eusociality. Nature 301: 59-62.

STRAND, M. R. 1986. The physiological interations of parasitoids with their hosts and their influence on reprodutive strategies, p. 97-136. In: J. WaAge \& D. Greathread (eds.). Insects Parasitoids. London, Academic Press, $680 \mathrm{p}$.

Strassamann, J. E.; D. C. Queller \& C. R. Hughes. 1988. Predation and the evolution of sociality in the paper wasp Polistes bellicosus. Ecology 69(5): 1497-1505.

Tauber, M. J. \& C. A. Tauber. 1976. Insect seasonality: diapause maintence, termination, and post diapause development. Annual Review of Entomology 21: 81-107.

Turillazzi, S. 1991. The Stenogastrinae, p. 74-98. In: K. G. Ross \& R. W. Matthews (eds.). The social biology of wasps. Ithaca, Cornell University Press, $678 \mathrm{p}$.

WCisLo, W. T. 1984. Gregarious nesting of a digger wasp as a 'selfish herd' response to a parasitic fly (Hymenoptera: Sphecidae; Diptera: Sarcophagidae). Behavioral Ecology and Sociobiology 15: 157160.

WCISLO, W. T. 1996. Parasitism rates in relation to nest site in bees and wasps (Hymenoptera: Apoidea). Journal of Insect Behavior 9(4): 643-656.

WCislo, W. T.; B. S. Low \& C. J. Karr. 1985. Parasite pressure and repeated burrow use by different individuals of Crabro (Hymenoptera: Sphecidae; Diptera: Sarcophagidae). Sociobiology 11(2): 115-125.

Wilson, E. O. 1971. The insect societies. Cambridge, Belknap Press, $548 \mathrm{p}$.

YANEGA, D. 1997. Demography and sociality in halictine bees (Hymenoptera: Halictidae), p. 293-315. In: J. C. ChOE \& B. J. CResPI (eds.). The evolution of social behavior in insects and arachnids. Cambridge, Cambridge University Press, $541 \mathrm{p}$.

ZAR, J. H. 1999. Biostatitical analysis (4th Ed.). New Jersey, Prentice Hall, $663 \mathrm{p}$.

ZuCCHI, R.; S. F. SAKAGAMI \& J. M. F. CAMARgO. 1969. Biological observations on a Neotropical parasocial bee, Eulaema nigrita, with a review on the biology of Euglossinae (Hymenoptera, Apidae). A comparative study. Journal of the Faculty of Science, Hokkaido University, Series VI, Zoology 17(2): 271-380. 